\title{
Far infrared Fourier transform spectrometer breadboard activities for the FORUM mission, ESA's 9th Earth Explorer
}

C. Pachot, B. Carnicero Domínguez, B. Sierk, F. Mariani, S. Riel, et al.

C. Pachot, B. Carnicero Domínguez, B. Sierk, F. Mariani, S. Riel, H. Oetjen, G. Rodrigues, S. Ratzsch, D. Redlich, W. Posselt, E. Alberti, C. Mastrandrea, C. Monte, Y. Montembeault, D. Smith, P. Ade, X. Durandi, M. Kolm, "Far infrared Fourier transform spectrometer breadboard activities for the FORUM mission, ESA's 9th Earth Explorer," Proc. SPIE 11852, International Conference on Space Optics — ICSO 2020, 1185224 (11 June 2021); doi: 10.1117/12.2599355

SPIE Event: International Conference on Space Optics - ICSO 2021, 2021, Online Only 


\section{International Conference on Space Optics-ICSO 2020}

Virtual Conference

30 March-2 April 2021

Edited by Bruno Cugny, Zoran Sodnik, and Nikos Karafolas
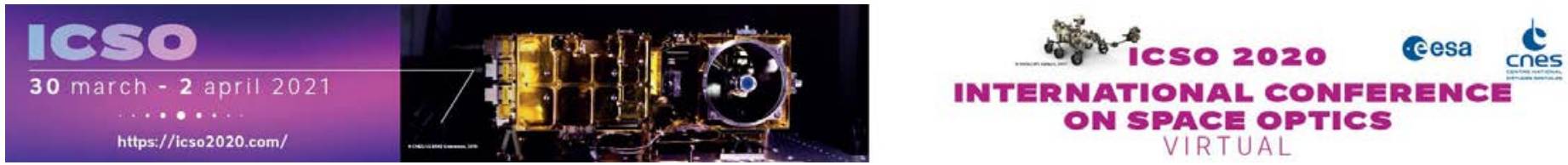

\section{Far infrared Fourier transform spectrometer breadboard activities for the FORUM mission, ESA's 9th Earth Explorer}

\section{Cesa isso procedings denes}




\title{
Far InfraRed Fourier Transform Spectrometer breadboard activities for the FORUM mission, ESA's 9th Earth Explorer
}

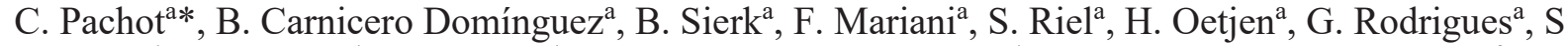

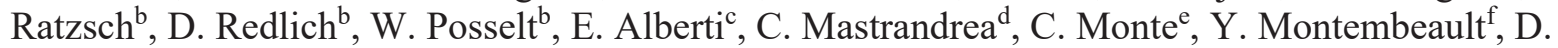

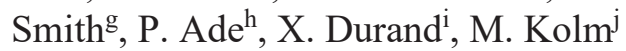 \\ ${ }^{\text {a }}$ European Space Agency/ESTEC, Keplerlaan 1, PO Box 299, 2200AG Noordwijk, The \\ Netherlands; ${ }^{b}$ OHB System AG, Manfred-Fuchs-Str. 1, D-82234 Weßling, Germany; ${ }^{\mathrm{c}}$ Micos \\ Engineering GmbH, Überlandstrasse 129, CH-8600 Dübendorf, Switzerland; ' ${ }^{2}$ EONARDO, Via \\ delle Officine Galileo, Campi Bisenzio (FI) 50013, Italy; ${ }^{\text {e }}$ Physikalisch-Technische Bundesanstalt \\ (PTB), Abbestrasse 2 - 12, D-10587 Berlin, Germany; ${ }^{\mathrm{f}}$ ABB Inc., 3400, Rue Pierre-Ardouin

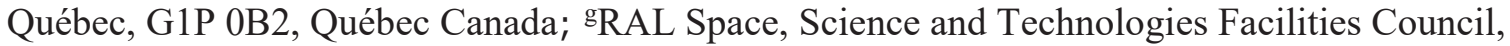 \\ Harwell Oxford, OX11 0QX, UK; ${ }^{h}$ Cardiff University, The Parade, Cardiff CF24 3AA, United \\ Kingdom; ${ }^{\mathrm{i}}$ Airbus Defence and Space, 31 Rue des Cosmonautes, 31400 Toulouse, France; ${ }^{j}$ Airbus \\ Defence and Space GmbH, Willy-Messerschmitt-Str. 1, 82024 Taufkirchen, Germany
}

\begin{abstract}
Significant progress has been made over the past years in the understanding of Earth's climate; however, there are still uncertainties in the modelling of atmospheric radiative processes. The main radiative processes relevant for climatological models are related to atmospheric water vapour, clouds, and surface emissivity across the full thermal infrared spectrum. Whilst the mid-infrared outgoing radiation is currently measured from space (e.g. IASI), there is a lack of spectrally resolved measurements over a significant portion of Earth's thermal emission in the Far-InfraRed (FIR) i.e. between 100 and $667 \mathrm{~cm}-1$ (15-100 micron).

FORUM (Far-infrared Outgoing Radiation Understanding and Monitoring) has been selected as the ESA's ninth Earth Explorer mission in September 2019. FORUM aims at measuring the Earth's Top-Of-Atmosphere emission spectrum from 100 to $1600 \mathrm{~cm}^{-1}$ (i.e. 6.25 to $100 \mu \mathrm{m}$ ). Two independent instrument concepts, based on a Fourier Transform Spectrometer, have been thoroughly studied in Phase A preparatory activities, including the development of a breadboard of the interferometer. In addition, critical technologies have been identified and subjected to comprehensive breadboard studies in order to mitigate development risks and to raise the Technology Readiness Level (TRL). This has led to the development and characterisation of several beamsplitter candidates, the measurement of the emissivity of the black coating for the on-board blackbody, the verification of noise and responsivity performance of pyroelectric detectors and the characterisation of the interferometer mechanism.
\end{abstract}

Keywords: Far Infrared, ESA Earth Explorer, FORUM, Fourier Transform Spectrometer, blackbody, diamond beamsplitter, pyroelectric detectors, interferometer

*charlotte.pachot@esa.int

\section{INTRODUCTION}

The European Space Agency's Earth Explorers, as part of ESA's Living Planet Programme, are research missions designed to address key scientific challenges while demonstrating breakthrough technology in the observation techniques. ESA selected two missions as candidates for implementation in the context of the 9th Earth Explorer call: the Far-infrared Outgoing Radiation Understanding and Monitoring (FORUM) mission and the Surface ocean KInematics Multiscale (SKIM) mission. These two missions were both subjected to feasibility studies, each carried out in parallel by two independent industrial consortia. At the User Consultation Meeting in Cambridge UK in July 2019 [1], at the end of 
phase A, the FORUM mission was recommended for implementation as the ESA 9th Earth Explorer mission. This recommendation was endorsed by the Programme Board for Earth Observation in September 2019. A summary of the motivations for the mission, with the scientific advances that are expected from these new measurements, is given in [2].

The FORUM phase A competitive studies have been led by Thales Alenia Space Ltd (UK) and Airbus Defence and Space (UK) as mission prime contractors, and OHB System AG and Airbus Defence and Space GmbH as instrument primes, respectively. Throughout this paper, the ADS team concept will be referred to as Concept A and the TAS team concept will be referred to as Concept B. This paper presents the results for the FORUM instrument of the work carried out in parallel by both industrial consortia in terms of critical technology development activities undertaken in phase A. The results provided here are representative of the end of the phase A study.

\section{MISSION CONCEPTS AND INSTRUMENTS DESIGN}

The FORUM space segment consists of a single satellite carrying two optical instruments: the FORUM Sounding Instrument (FSI) and the FORUM Embedded Imager (FEI). FORUM performs continuous step-and-stare nadir-looking observations of spectrally-resolved top of atmosphere (TOA) radiances in the mid-infrared (MIR) and far-infrared (FIR) regions of the spectrum. The primary instrument is the FSI, a single-pixel infrared-atmospheric-sounder Fourier Transform Spectrometer (FTS) sampling the electromagnetic spectrum from $100 \mathrm{~cm}-1$ to $1600 \mathrm{~cm}-1$ (100 $\mu \mathrm{m}$ to 6.25 $\mu \mathrm{m})$ with a spectral resolution better than $0.5 \mathrm{~cm}-1$. The FSI single spatial sample is located within the footprint of the FEI, an infrared imager observing in a single spectral band centered at $10.5 \mu \mathrm{m}$ with a bandwidth of $1.5 \mu \mathrm{m}$ and used for scene heterogeneity determination. Both instruments share several common units, such as the entrance aperture, the pointing mechanism, the thermal and mechanical framework and the radiometric calibration devices (see [3] for more details on the mission).

The instruments architectures resulting from both industrial studies are very similar and can be summarized in a single block diagram, shown in Figure 1 (for more details see [4]).

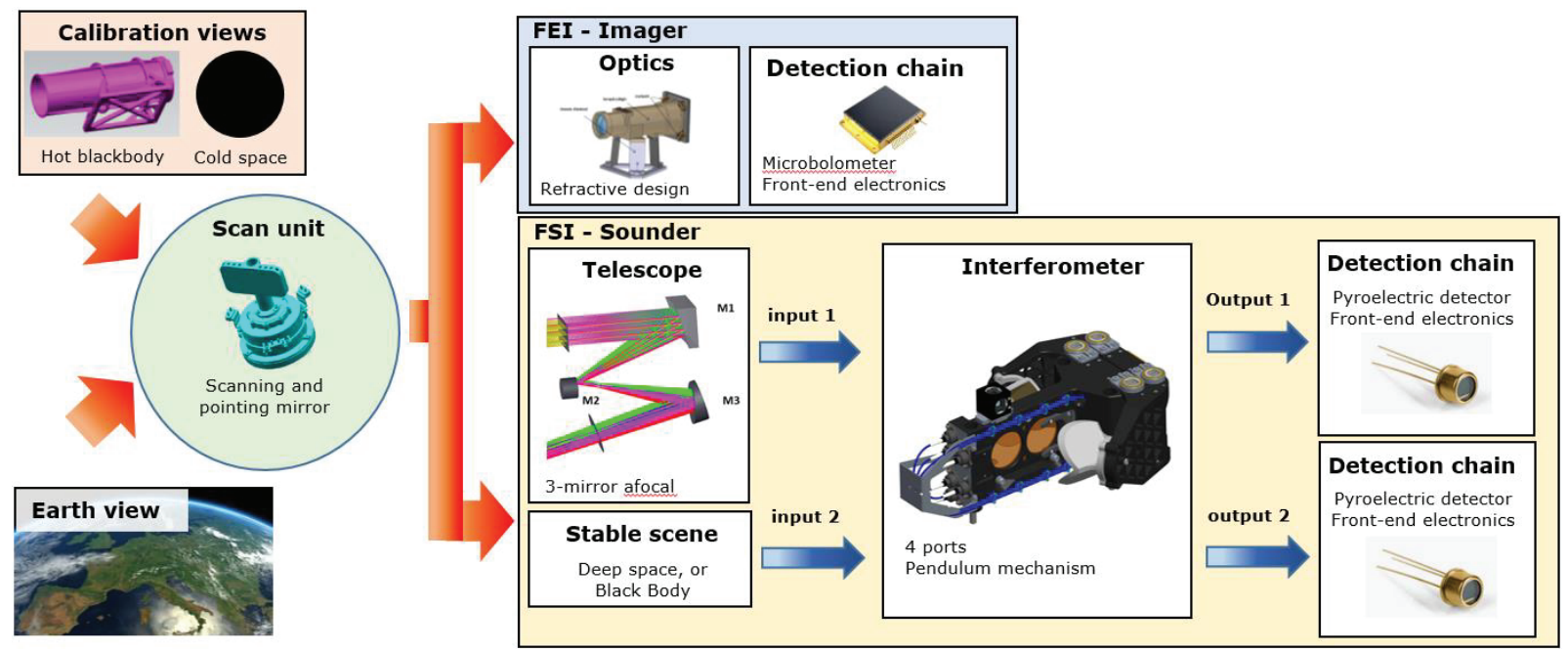

Figure 1. Payload functional black diagram

The main subsystems are:

- Calibration (blackbody/deep space) and Earth ports and associated baffles: the instruments perform science acquisitions using the Earth port and calibration acquisitions using either deep space or an on-board blackbody cavity. The on-board calibration blackbody has been identified as a critical element early in phase A and subjected to a pre-development activity. While being a key subsystem for the radiometric performance, the black coatings emissivity characteristics were not available over FORUM spectral range 
and had to be characterised. Due to stringent radiometric requirements, both consortia defined slightly different in-flight radiometric calibration strategies, alternating Earth views and calibration acquisitions, with a loss in coverage less than $20 \%$.

- Pointing and scanning unit common to both instruments: this is based on 1 or 2 mirrors to select the different ports (Earth, deep space or calibration blackbody) and to maintain a stable ground pointing during the simultaneous acquisitions of the FSI interferogram and the FEI images.

- FSI instrument: this is composed of a front telescope, a four-port interferometer (including corner cubes, beasmplitter and metrology system), focusing optics and a detection chain based on pyroelectric detectors, operated at ambient temperature, and associated electronics. Three elements have been identified as critical in this instrument: the detection chain for which a low noise design and characterization over the FORUM spectral range was needed, the interferometer as key subsystem of the instrument and the beamsplitter with a material choice limited by the FORUM wide spectral range in the mid to far infrared. Feasibility of the detection chain, interferometer and beamsplitter within the FORUM needs had to be studied early in the phase A to mature technology and de-risk the project.

- FEI instrument: it is composed of refractive optics, a bandpass filter, an ambient temperature microbolometer detector and associated electronics. It shares the pointing mechanism and the calibration with the FSI instrument.

An Instrument Control Unit (ICU) provides control and monitoring of the FSI, the FEI, and the pointing mechanism. The ICU is the interface for power and data between the Front End Electronic (FEE) and the platform. The interferometer has additionally a dedicated Interferometer Control Electronics (ICE). The ICU and the ICE are both mounted inside the platform and connected to the instrument modules via harnesses and optical fibres.

\section{BEAMSPLITTER}

In early Phase $\mathrm{A}$, the beamsplitter was identified as a critical component owing to the need to demonstrate transmission and reflection properties in the FORUM spectral range compatible with the radiometric and spectral requirements. In this extended infrared spectral range, not so many materials would fit FORUM needs: several options of beamsplitters were then investigated: CVD diamond beamsplitter plate, Mylar pellicle, and polypropylene pellicle. Such beamsplitters with the thickness and diameter required for FORUM have never been manufactured and flown. Dedicated pre-development activities were initiated to demonstrate the manufacturing feasibility and the compatibility of these components with the space environment.

Table 1. FORUM interferometer beamsplitter solutions both baseline and back-ups (Concepts A and B).

\begin{tabular}{|l|c|c|}
\hline Beamsplitter & Concept A & Concept B \\
\hline \multirow{3}{*}{ Baseline } & Dual-wedged diamond plate & Dual-wedged diamond plate \\
& Germanium coating on $1^{\text {st }}$ side & Germanium coating on $1^{\text {st }}$ side \\
& Anti-Reflection coating on $2^{\text {nd }}$ side & Bare diamond on $2^{\text {nd }}$ side \\
& Option: Anti reflection coating on $2^{\text {nd }}$ \\
& Dual polypropylene membrane & Single polypropylene membrane \\
Back-ups & Dual Mylar membrane & Single Mylar membrane \\
\hline
\end{tabular}

\subsection{Baseline design - Diamond plate beasmplitter}

The selected baseline beamsplitter is a dual-wedged diamond plate. Diamond is a good candidate material showing a quite high and relatively constant transmission over the full FORUM spectral range. The splitting interface (front face) between the two arms is enhanced by applying a germanium coating on the diamond substrate. The rear face of the beamsplitter is either bare diamond (introducing Fresnel losses in the order of $15 \%$ in transmission due to the diamond high-optical refractive index) or an antireflective treatment improving the overall transmission. As the FORUM spectral 
range is very wide, it was difficult to find a classic dielectric antireflective coating. Therefore, the implementation of microstructures on diamond has been studied as a means to enhance the transmission. The feasibility of such diamond beamsplitter (germanium coated on one side and microstructured on the other side) had already been demonstrated in the frame of NASA's OSIRIS-REX mission [5], but for a smaller beamsplitter size (38 $\mathrm{mm}$ in diameter versus around 60-70 $\mathrm{mm}$ necessary for FORUM FSI). Within the pre-development activities in Phase A, germanium-coated diamond samples, microstructured diamond samples and full-size wedged and coated diamond beamsplitters were manufactured and tested for both consortia.

The different steps that the samples underwent during the Phase-A pre-development activities are summarised in Table 2.

Table 2. Beamsplitter baseline phase A pre-development activities summary table - Concept A and B.

\begin{tabular}{|l|c|c|c|}
\hline & $\begin{array}{c}\text { Germanium-coated } \\
\text { diamond samples }\end{array}$ & $\begin{array}{c}\text { Microstructured diamond } \\
\text { samples }\end{array}$ & $\begin{array}{c}\text { Full-size wedged } \\
\text { diamond plate }\end{array}$ \\
\hline Manufacturing & $\sqrt{ }$ & $\sqrt{ }$ & $\sqrt{ }$ \\
\hline Coating & $\sqrt{ }$ & $\sqrt{ }$ & $\sqrt{ }$ \\
\hline Performance testing & $\sqrt{ }$ & NA & $\sqrt{ }$ \\
\hline $\begin{array}{l}\text { Transmission/Reflection over full } \\
\left.\text { FSI spectral range [100-1600 } \mathrm{cm}^{-1}\right]\end{array}$ & NA & & \\
\hline $\begin{array}{l}\text { WFE measurement (before and } \\
\text { after coating) }\end{array}$ & $\sqrt{ }$ & NA & NA \\
\hline Environmental testing & $\sqrt{ }$ & NA & NA in this phase \\
\hline Adhesion test & $\sqrt{ }$ & & \\
\hline TVAC test & & & \\
\hline
\end{tabular}

Diamond samples of size smaller than the final beamsplitter were coated with germanium of different thicknesses and characterized for transmission and reflection performance before environmental tests (see 4RT performance in Figure 5.21 left) over the full FORUM spectral range. The same diamond samples were also successfully thermally cycled in vacuum.

Additional diamond samples were manufactured with microstructures and the transmission improvement, with respect to bare diamond interface, was characterised over the full FORUM spectral range; results are reported in Figure 2. The effect, in the range of $5-15 \%$ depending on the wavenumber, improves the overall radiometric (noise) performance of the instrument.
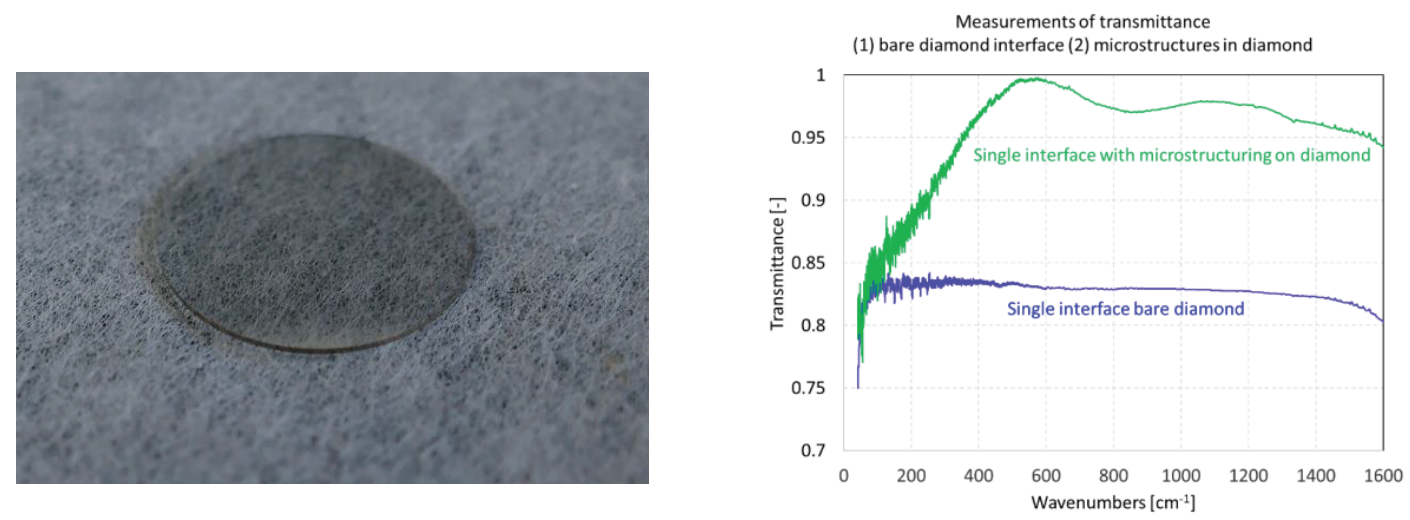

Figure 2. (Left) Uncoated diamond sample, (Right)Transmittance of single interface bare diamond compared to bare diamond including microstructuring versus wavenumber - Concept A 
In addition to the diamond samples (small size), full-scale wedged-diamond beamsplitters have been manufactured, coated and tested by Concept B. Figure 3 shows a picture of a true-sized wedged diamond beamsplitter manufactured during the pre-development activities. It demonstrates the feasibility of manufacturing and coating a wedged-diamond substrate of the size needed for FORUM (60-80 $\mathrm{mm}$ in diameter). Figure 4 right reports the compliant WaveFront Error (WFE) performance measured on the full-scale diamond plate after coating and the beamsplitter efficiency (4RT) Figure 4 left - based on measured reflection and transmission data.
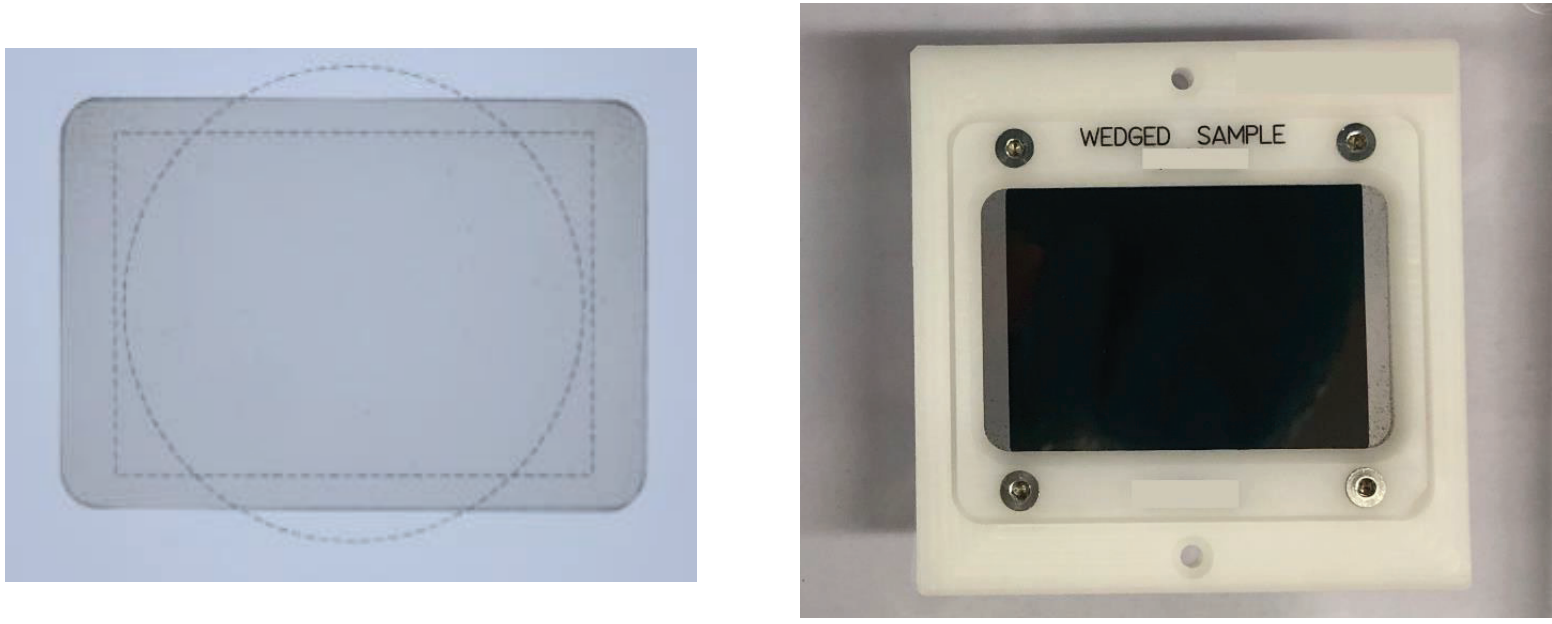

Figure 3. FSI interferometer full size wedged diamond beamsplitter breadboard uncoated (Left) and after Germanium coating (Right) - Concept B
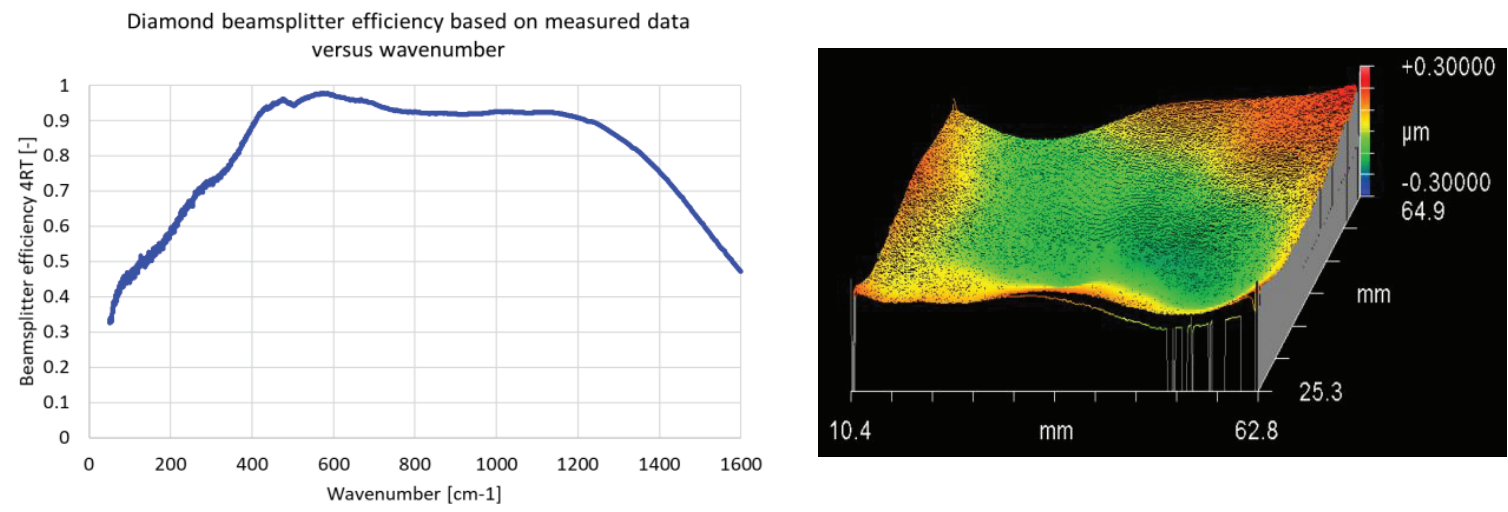

Figure 4 (Left) FSI diamond beamsplitter Efficiency based on measurement (Concept A), (Right) Ge-coated diamond WFE measurement in reflection on full scale sample) - WFE measured $<100 \mathrm{~nm}$ rms (Concept B)

\subsection{Alternative designs - Mylar and Polypropylene beasmplitter}

Membranes beamsplitters made of Polypropylene or Mylar (see Figure 5) have been studied as back-up beamsplitter solutions for the FORUM FSI. Mylar membranes used in the FORUM spectral range have flight heritage as polarising beamsplitter on the Cassini Composite InfraRed Spectrometer (CIRS), and ground heritage on the REFIR-PAD instrument. Compared to diamond, the Mylar and Polypropylene exhibit absorption features in the FORUM spectral 
range which would lead to non-compliance to the FSI noise requirement in the absorption bands. In addition, the membranes are sensitive to mechanical and thermal stresses.

Both membrane options have a germanium coating as splitting surface. Both Polypropylene and Mylar beamsplitters have been manufactured and coated with Germanium within the phase A pre-development activities. These have been mechanically mounted in design-representative stainless steel or aluminum mounts, and transmission and reflection measurements over the full FORUM spectral range have been performed under vacuum. WFE measurements have also been performed.

The Polypropylene substrate with germanium coating were not successful: compared to predictions, these showed significantly lower beasmplitter efficiency and a much higher WFE, not acceptable for the FORUM required modulation efficiency to meet the stringent radiometric performance requirements. Despite attempts to improve the manufacturing process, with the current performance Polypropylene is not considered a viable solution for FORUM. While the Mylar option, in turn, achieves reasonable WFE performance, the germanium depositions show non-homogeneities which worsen over time, degrading the expected beasmplitter efficiency and WFE. Further investigations and improvements would be needed with Mylar coating to be able to meet FORUM needs. This is considered as an alternative to diamond based solution.
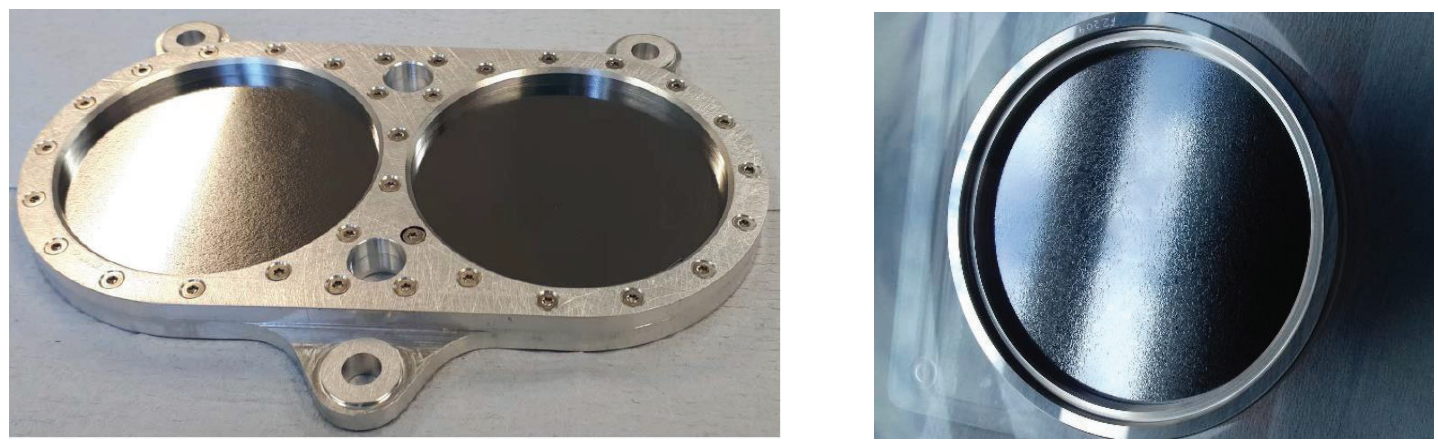

Figure 5. FSI Beamsplitter back-up solutions: (Left) Concept A Polypropylene beasmplitter, (Right) Concept B Mylar beamsplitter. (Courtesy of Cardiff University)

\section{ON-BOARD BLACKBODY}

FORUM embarks an on-board blackbody source to perform in-flight radiometric calibration. The instruments are calibrated in flight using a deep-space view and a blackbody maintained at instrument temperature (e.g. 293 K), with the temperature being monitored to provide a known reference radiance. The calibration blackbody is implemented at the entrance of the instruments following the same path as the Earth view, with only a different angle of incidence at the pointing mirror. The calibration blackbody baseline for both concepts is based on well-proven black-coated cavity geometries, with a design allowing the emissivity requirement to be met $(>0.995$ for most of the FORUM spectral range).

The black coating baseline has been selected based on performance, stability and heritage. The outcome of the trade-off and pre-developments of Phase A converged towards the selection of the high emissivity paint Mankiewicz Nextel 811 21 which has heritage on-ground and in flight. The emissivity of several samples of Nextel 811-21 in the FORUM spectral range up to $100 \mu \mathrm{m}$ has been measured in ambient and in vacuum at the Physikalisch-Technische Bundesanstalt (PTB) facilities in Berlin together with other possible black coating candidates (Figure 6). 

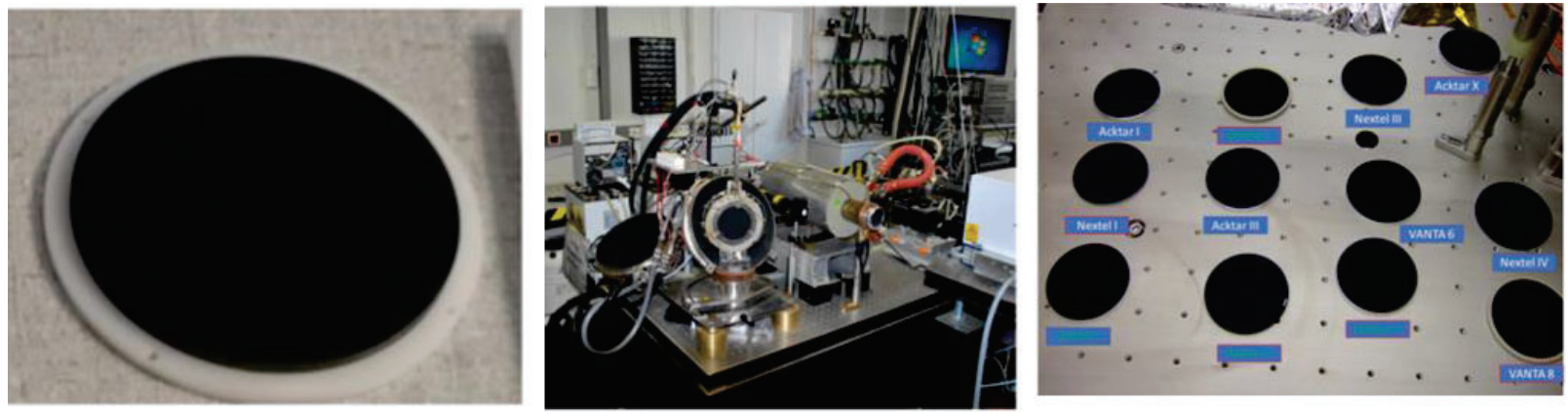

Figure 6 From left to right: (1) Black-coated sample for emissivity measurement Nextel 811-21, (2) emissivity measurement set-up in air at PTB, (3) black-coated samples prepared for environmental tests.

Nextel black coating heritage includes, among others, the blackbody cavity of the High Resolution Dynamics Limb Sounder (HIRDLS) instrument on the NASA EOS Chem satellite. Other potential black coating candidates have better performance but less heritage: in particular, the Vanta S-VIS, S-IR from Surrey Nanosystems, and Deep Sky Black from Equinox have been considered as options. The emissivity of all the above mentioned black coating options has been characterised over the complete FORUM spectral range from $6 \mu \mathrm{m}$ to $100 \mu \mathrm{m}$. The baseline and optional black-coated samples underwent environmental testing, including adhesion test, thermal vacuum cycling and humidity. Additionally, the deposition process repeatability for the Nextel black coating has been assessed within the pre-development activity, with two samples from two different batches being tested. The results (see Figure 7) prove a stable performance.
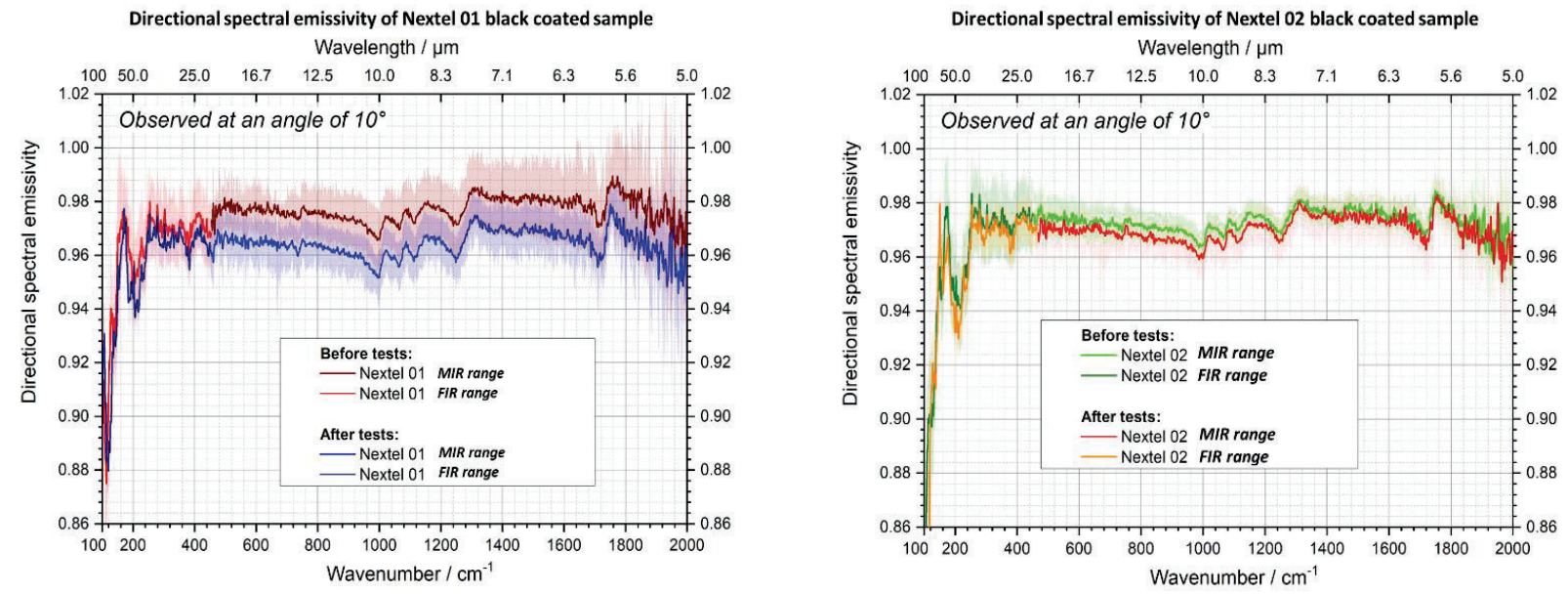

Figure 7 Nextel 811-21 black coated sample emissivity measurement results before and after environmental testing for 2 different samples manufactured from 2 batches - Concept A and B. (Courtesy of PTB).

The blackbody geometry has been iterated based on some blackbody performance simulations using the STEEP software. Several trials of geometry and black coating types have been performed in order to identify the best compromise. A Nextel coated cylindrical cavity geometry has been selected as baseline allowing to reach the emissivity requirement. The cavity model has been refined, to include a thermal model and thermal environment simulations to assess the temperature gradient over the cavity and to evaluate the impact on radiometric performance. This analysis confirmed the compliance of the blackbody design to the requirements.

Concept A design includes a phase changing cell for absolute calibration in orbit of the temperature sensors, to reduce the contributor in the overall Absolute Radiometric Accuracy budget due to the sensors long term drift. A predevelopment activity has been completed in phase A, by building a representative mock-up and testing the repeatability of the melting point. The results confirm a repeatability well within the requirement of $10 \mathrm{mK}$, as shown in Figure 8 . 

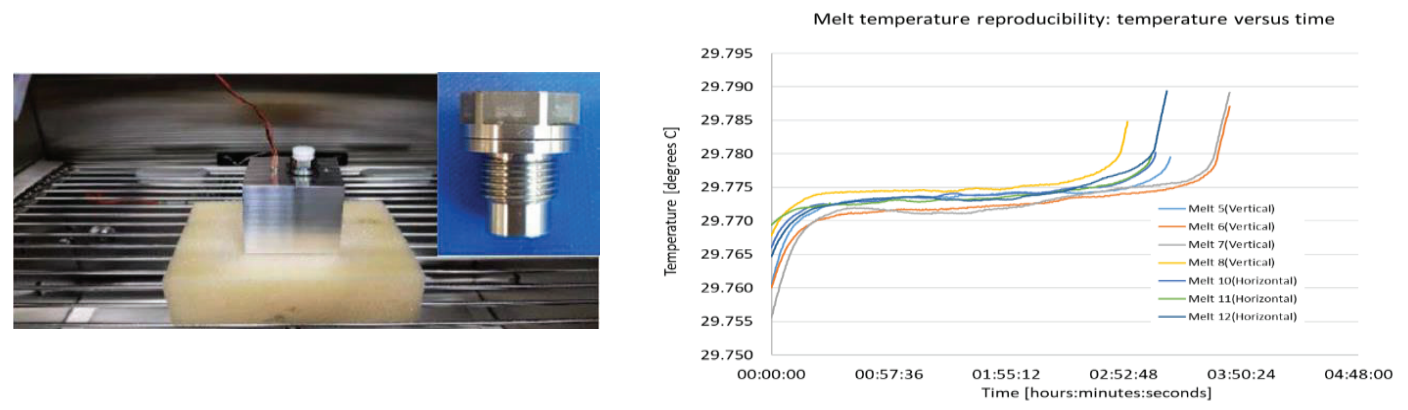

Figure 8 (Left) Phase change cell mock-up for plateau repeatability measurement, (Right) Melt temperature reproducibility within $10 \mathrm{mK}$ measurement results - Concept A

In in-flight operations, the temperature of the blackbody, which is maintained at floating temperature (isothermal with the instrument), is temporarily increased to around $30^{\circ} \mathrm{C}$ to reach the melting point of the phase-change cell and perform the calibration of the thermal sensors.

Concept B does not include an on-board phase change cell device for recalibrating the temperature sensor of the blackbody in-flight. The here applied Platinum Resistance Thermometer (PRT) sensors are known for being sensitive to vibrations and thermal cycling. The additional errors in the Absolute Radiometric Accuracy budget are accounted for and some measures have to be taken to limit the contribution:

- Temperature variations to be tracked to estimate the range of the PRT hysteresis

- Possible use of on-ground reference PRTs that would experience the same temperature variations

- Maintain the temperature in a small range during Assembly Integration and Test, delivery, and pre-flight to minimize the thermo-elastic effects

\section{DETECTION CHAIN}

The FORUM Sounder Instrument detection chain is located at each of the two outputs of the interferometer (see Figure 1), after the focusing optics, and it comprises the detectors and the electronics. The detectors convert the optical interferograms into an electrical signal, which is then sampled by the electronics and formatted into digital data to be sent to the Instrument Control Unit. The two detection chains are electrically and functionally independent.

The selected detectors are based on pyroelectric technology. A pyroelectric crystal exhibits a temperature dependent spontaneous electrical polarisation. Thanks to this property, temperature changes result in a change in the electric field at the two faces of the crystal that can be measured. This allows the detection of thermal infrared signals, as in the case of the FSI interferograms. The pyroelectric crystal used for FORUM detectors is Deuterated L-alanine Dopped Triglycine Sulphate (DLaTGS) a material offering very high sensitivity in the infrared spectral range. The crystal is grown as a wafer and polished into a thin element, with electrodes deposited on the opposite faces. The surface of the crystal exposed to infrared light, is blackened to enhance its absorption. The element is mounted in a hermetically sealed industry-standard electronic package, with a diamond window to admit radiation. The baseline detectors for both concepts are provided in Table 3. One of the advantage of such detector technology is that the detectors are operated at ambient temperature throughout the complete FORUM spectral range up to 100 microns, not requiring any cryocoolers. 
Table 3. Pyroelectric detectors used in the detection chain for the FSI Concepts A and B.

\begin{tabular}{|l|l|l|}
\hline & Concept A & Concept B \\
\hline Baseline detector & $\begin{array}{l}\text { LEONARDO's DLaTGS pyroelectric } \\
\text { detector 99 series standard }\end{array}$ & $\begin{array}{l}\text { LEONARDO's DLaTGS pyroelectric } \\
\text { detector 106 series standard }\end{array}$ \\
\hline Window/lens material & Diamond & Diamond \\
\hline Detector sensitive area & $2 \mathrm{~mm}$ & $2 \mathrm{~mm}$ \\
\hline Operation mode & Voltage mode & Voltage mode \\
\hline
\end{tabular}

The noise and the responsivity properties of several DLaTGS detectors were characterised in laboratory environment within the frame of the Phase A pre-developments activities. In the study for Concept A, absolute measurements were performed for comparison with the datasheet from manufacturers, while the pre-development for Concept B provided a relative comparison of the performances between the different detectors. The setup built for the detection chain predevelopments in both studies are shown in Figure 9, where the radiation source (blackbody), the detector and the electronics can be identified easily.

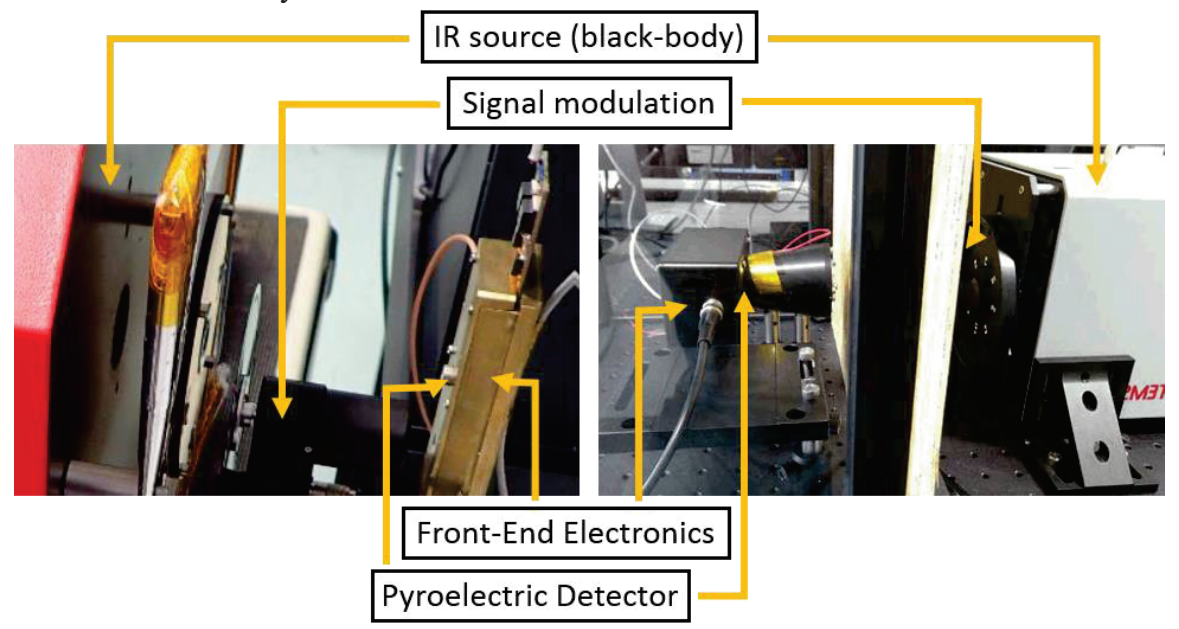

Figure 9. The setups built for the detection chain pre-developments activities for responsivity and noise characterisation of the pyroelectric detectors, for Concept A (left) and B (right).

Some results for the responsivity of the two baseline detectors are shown in Figure 10. The results for responsivity and noise are in line with the values provided by the detector suppliers datasheets for both baseline detectors. 

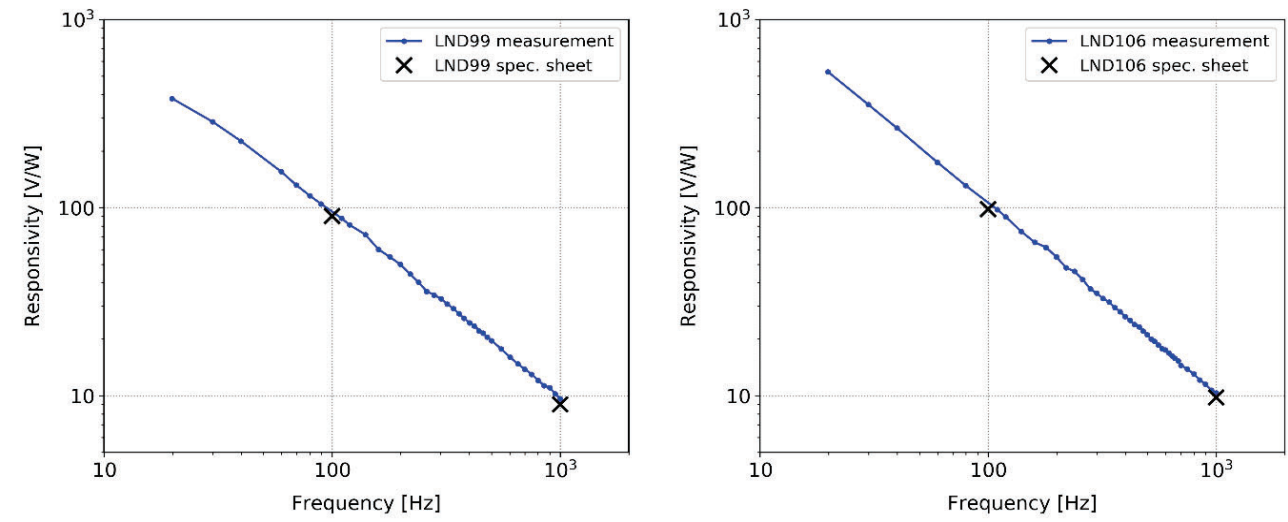

Figure 10. Results from the detection chain pre-development for the detectors considered as baseline in Concept A (left) and B (right).

The pre-development activities also focused on comparing and providing quantitative data from two different operation modes of the detector, voltage-mode and current-mode. The voltage-mode is the standard mode of operation, and the measurements within the pre-development activities confirmed the manufacturers experience and model. The investigations on the current-mode performance did not give conclusive results on the consistency between measurements and model.

\section{INTERFEROMETER}

A double-pendulum mechanism was selected for the interferometer in both concepts. This choice provides sufficient stroke and beam diameter with a simple configuration, and is robust to environmental loads. In the pendulum mode of operation, the corner cubes located at the tip of both arms move in opposite directions and the resulting Optical Path Difference (OPD) between the two optical path produces the interferogram.

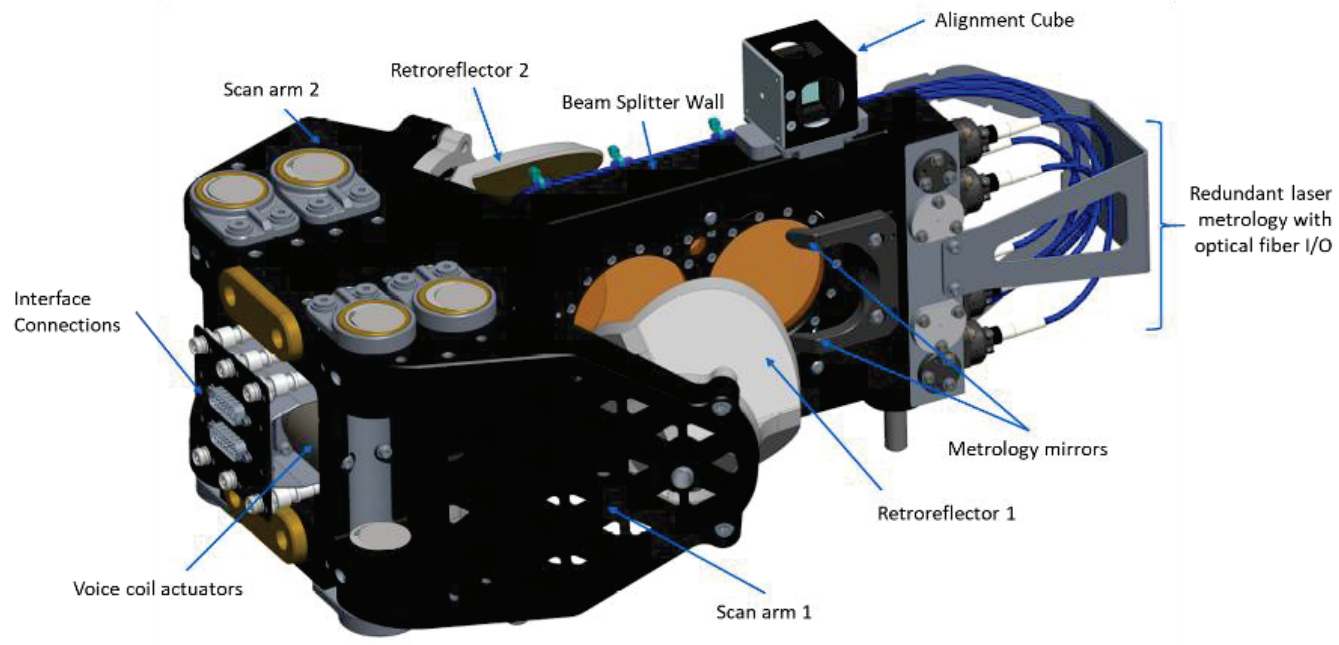

Figure 11. FORUM FSI Interferometer breadboard sketch - Concept A. (Courtesy ABB). 


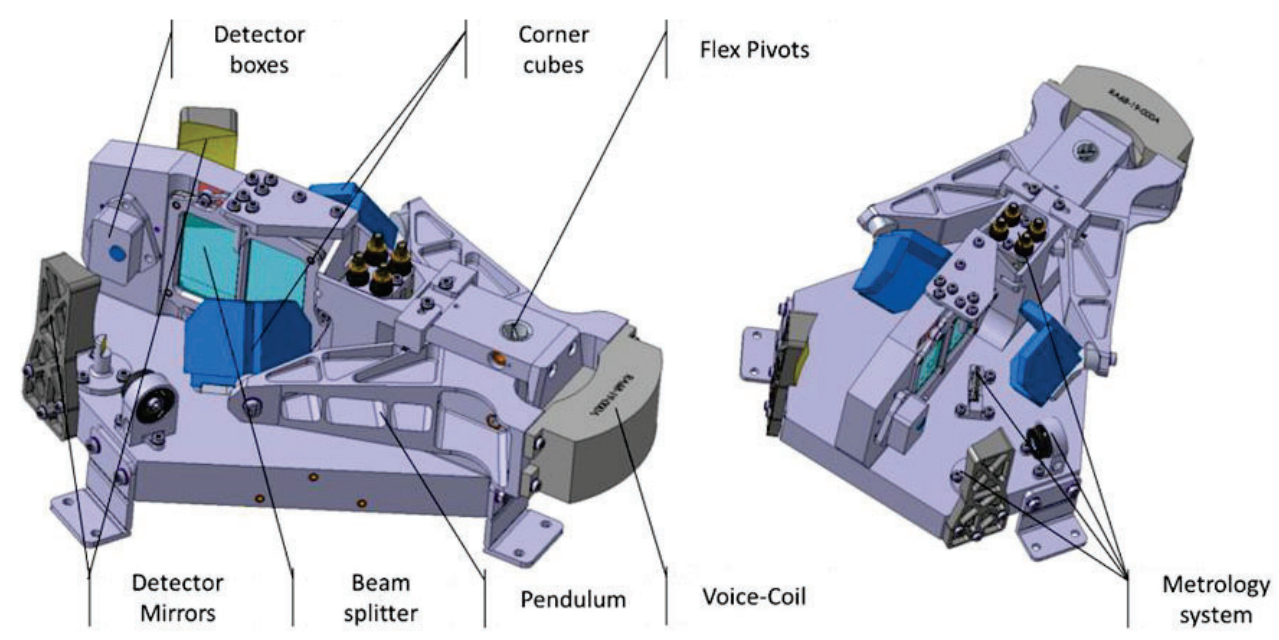

Figure 12. FORUM FSI interferometer sketch - Concept B. (Courtesy OHB)

Concept A has heritage from commercial ground-based interferometers, with thousands of units currently operational with the same double pivot mechanism as the ones proposed for FORUM (see Figure 11). Moreover, a prototype of the double pivot mechanism, smaller in size but representative of FORUM's, has undergone space qualification campaign in the frame of the Large Aperture Dual Pivot (LADP) development. Concept B proposes to use a single pivot mechanism (see Figure 12), smaller in size but fully representative of the ones employed in the double-pendulum FTS instruments for the Atmospheric Chemistry Experiment (ACE)-FTS onboard of Science-Satellite (SciSat), and the TANSO-FTS on GOSAT. The interferometer mechanisms of both concepts are considered feasible and with proven heritage. However, pre-development activities have been performed in phase A to demonstrate the required performance for the specific FORUM design in the relevant environment, and an interferometer mechanism breadboard has been designed, manufactured, aligned and characterised.

The actuation principle involves a voice-coil (a double-coil for redundancy), which imposes the rotation of the two arms around the flexible pivot hinges. This configuration provides large rotations around a fixed axis, with high stiffness and virtually no friction even without lubrication.

Figure 13 shows an interferometer breadboard developed by ABB under a co-funding contract of the Canadian Space Agency, which includes the same mechanism as the one proposed for FORUM Concept A.

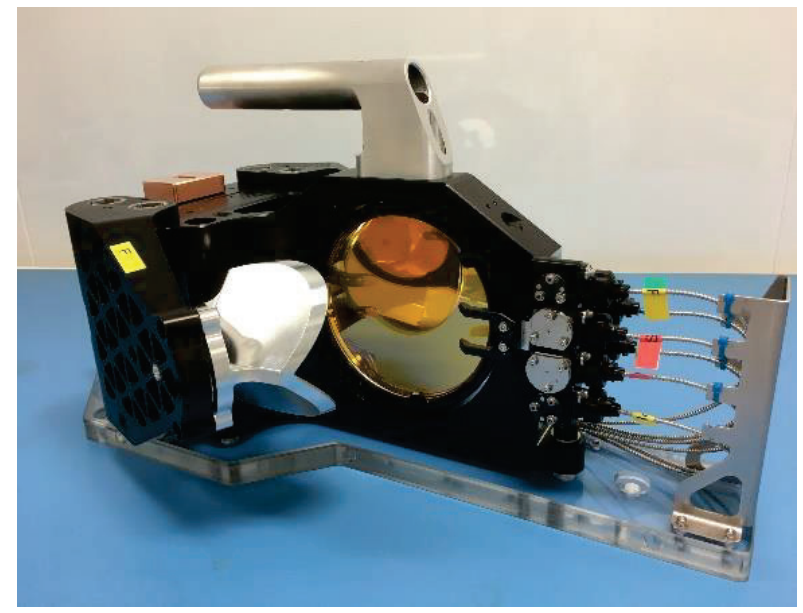

Figure 13. Large aperture interferometer breadboard developed by ABB partially funded by the Canadian Space Agency. (Courtesy $\mathrm{ABB})$ 
One of the requirements for the interferometer mechanism is that sampling speed instabilities shall remain lower than $1 \%$ during science acquisition. Analysis have confirmed that the interferometer design meets this requirement for both concepts. During the phase A pre-development activities, a breadboard for Concept B has been manufactured, aligned and tested - Figure 14. The speed stability and micro-vibrations tests have been successfully performed.

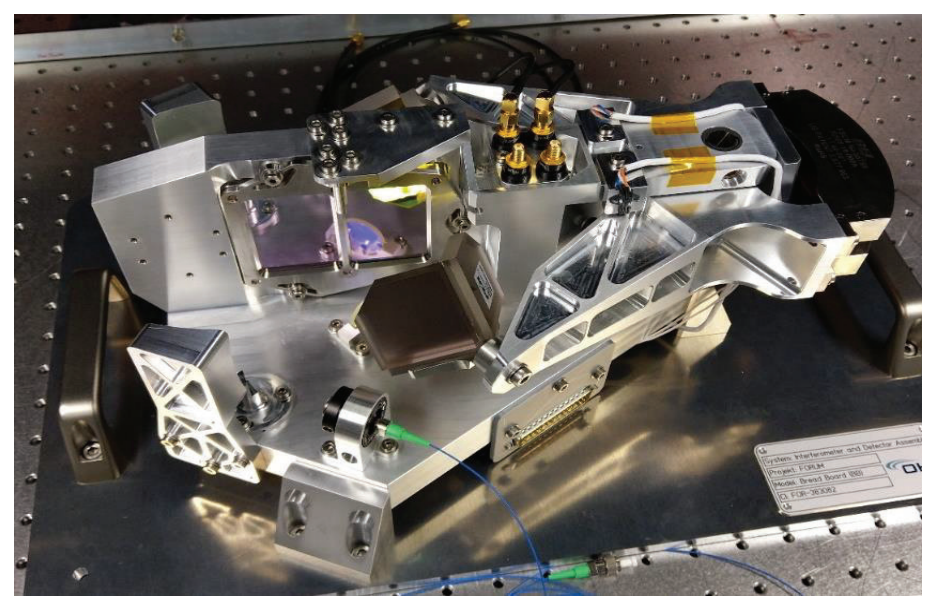

Figure 14. FORUM Interferometer mechanism breadboard Concept B. (Courtesy of OHB).

\section{CONCLUSION}

The FORUM industrial studies in the frame of the ninth Earth Explorer activities were accompanied by a comprehensive pre-development programme for critical components. In this paper, we presented the scope and results of the breadboard activities. First laboratory test results and critical components feasibility have been presented for the blackbody, the detection chain and the beamsplitters candidates over the full FORUM spectral range. The test results indicate that the challenging radiometric needs for FORUM can be met.

\section{ACKNOWLEDGEMENT}

The authors would like to thank all industrial partners involved in the preparatory activities, especially Airbus UK, Airbus GmbH, Thales Alenia Space UK and OHB, the scientific institutions and the Mission Advisory Group (MAG), in particular Luca Palchetti (Consiglio Nazionale delle Ricerche) and Helen Brindley (Imperial College London). 


\section{REFERENCES}

[1] Report for Mission Selection: FORUM, ESA-EOPSM-FORM-RP-3549 issue 01, 21 June 2019, European Space Agency, Noordwijk, The Netherlands, https://esamultimedia.esa.int/docs/EarthObservation/EE9-FORUM-RfMSESA-v1.0-FINAL.pdf, 2019

[2] Palchetti, L., Brindley, H., Bantges, R., Buehler, S. A., Camy-Peyret, C., Carli, B., Cortesi, U., Del Bianco, S., Di Natale, G., Dinelli, B. M., Feldman, D., Huang, X. L., C.-Labonnote, L., Libois, Q., Maestri, T., Mlynczak, M. G., Murray, J. E., Oetjen, H., Ridolfi, M., Riese, M., Russell, J., Saunders, R. and Serio, C., FORUM: unique farinfrared satellite observations to better understand how Earth radiates energy to space, Bulletin of the American Meteorological Society, 2020, pp. 1-52, https://doi.org/10.1175/BAMS-D-19-0322.1

[3] Bernardo Carnicero Domínguez et al., The far-infrared outgoing radiation understanding and monitoring (forum) mission. Esa's 9th earth explorer, IGARSS 2020

[4] Charlotte Pachot, Bernardo Carnicero Dominguez, Hilke Oetjen, Bernd Sierk, Flavio Mariani, Stefanie Riel, Gonçalo Rodrigues, Miguel Copano, Asier Martínez Carou, Luca Palchetti, Magdalena Lippa, Philippe Giaccari, Carmine Mastrandrea, Gaetan Perron, Corneli Keim, Manfred-Georg Kolm, "The infrared Fourier transform spectrometer and the infrared imager instrument concepts for the FORUM mission, ESA's 9th Earth Explorer," Proc. SPIE11530, Sensors, Systems, and Next-Generation Satellites XXIV, 115300D (20 September 2020); doi: $10.1117 / 12.2570867$

[5] Christensen et al., The OSIRIS-REX Thermal Emission Spectrometer (OTES) Instrument, 2018, https://ink.springer.com/article/10.1007/s11214-018-0513-6 